\title{
Experimental Genetics
}

National Cancer Institute

\section{Source}

National Cancer Institute. Experimental Genetics. NCI Thesaurus. Code C18811.

Basic research in the field of genetics. 